\title{
Energy Efficient Data Processing In Visual SENSOR NETWORK
}

\author{
Harish. H. Kenchannavar ${ }^{1}$, Sushma. S. Kudtarkar ${ }^{2}$, U. P. Kulkarni ${ }^{3}$ \\ ${ }^{1}$ Dept of Computer Science \& Engineering Gogte Institute of Technology, \\ Belgaum India \\ harish_14@rediffmail.com \\ ${ }^{2}$ Dept of Computer Science \& Engineering Gogte Institute of Technology, \\ Belgaum India \\ sushmakudtarkar@gmail.com \\ ${ }^{3}$ Dept of Computer Science \& Engineering SDMCET, Dharwad India \\ upkulkarni@yahoo.com
}

\begin{abstract}
Identifying moving objects from a video sequence is a fundamental and critical task in many computervision applications. After the images are captured they must be processed and then sent to the server. In this paper we characterize the energy consumption of a visual sensor network testbed. Each node in the testbed consists of a "single-board computer", equipped with a network card and a webcam. We assess the energy consumption of activities representative of the target application (e.g., perimeter surveillance) using a benchmark that runs (individual and combinations of) "basic" tasks such as processing, image acquisition, and communication over the network. In our characterization, we consider the various hardware states that the system switches through as it executes these benchmarks, e.g., different radio modes (sleep, idle, transmission, reception), and webcam modes (off, on, and acquiring image) using Matlab Sensor Node and Lifetime simulator. We report the energy utilized by each frame during transmission at the server. Here we can analyze the energy consumed with processing and without processing of video frames.
\end{abstract}

\section{KEYWORDS}

Surveillance, Motion Detection, Energy Consumption, Reconstruction.

\section{INTRODUCTION}

Visual sensor networks are networks of wireless camera-nodes, where the camera-node consists of the image circuitry, a processor, and a wireless transceiver. The network generally consists of the cameras themselves, which have some local image processing, communication and storage capabilities, and possibly one or more central computers, where image data from multiple cameras is further processed and fused (this processing may, [1],[2] however, simply take place in a distributed fashion across the cameras and their local controllers). Visual sensor networks also provide some high-level services to the user so that the large amount of data can be distilled into information of interest using specific queries. Visual sensor networks suitable for use in 
applications where temporary monitoring is needed and in applications that require fast deployment and removal of the camera network.

Cameras are directional in their field of view and they capture a large amount of visual information which may be partially processed independently of data from other cameras in the network. There are different features of visual sensor network such as energy limitations, data processing, resistance to node failure, scalability, heterogeneity, Quality of Service (QoS), flexible architecture to support heterogeneous applications, multimedia coverage out of which, we consider only two issues in this paper i.e data processing and energy consumption.

Data processing: Data collected by the sensor nodes that lie in proximity to each other may contain a high level of spatial and temporal redundancy [3]. Local data processing (through data aggregation or data fusion) reduces the amount of data that need to be transmitted back to the data sink, thereby providing the application with high-level data representations that qualitatively satisfy the application's requirements. Using a background subtraction technique we can reduce the size of the image and the energy required to transmit each pixel.

Energy Consumption: In the absence of promising energy-scavenging technologies that would provide constant energy supplies for the sensor nodes, batteries are the most commonly used sources of energy [4]. Energy is thus a scarce resource, and it presents a basic limiting factor for the node's lifetime. Thus, intelligent polices for the efficient utilization of the energy resources are needed. In this paper, we have considered the energy consumed during subtraction, transmission and reconstruction.

\section{BACKGROUND}

Visual sensor networks are based on several diverse research fields, including image/vision processing, communication and networking, and distributed and embedded system processing. Due to its interdisciplinary nature, the research directions in visual sensor networks are numerous and diverse. In the following sections we present an overview of the ongoing research in several areas vital to visual sensor networks: Sensor localization and camera calibration in distributed camera sensor networks by A. Barton-Sweeney, D. Lymberopoulos, and A. Savvides, do not provide for fully automatic camera calibration methods, but instead they point out the difficulty of finding appropriate network configurations that can ease the calibration process [5]. Panoptes: scalable low-power video sensor networking technologies by E. Kaiser, M. Shea, W.-C. Feng, and L. Bavoil proposed the first video-based sensor network systems, and it includes video sensors built from COTS and software that supports different functions including capture, compression, filtering, video buffering and streaming [5]. Balancing computational and transmission power consumption in wireless image sensor networks by L. Ferrigno, S. Marano, V. Paciello, and A. Pietrosanto, aims at finding the most suitable compression method that provides the best compromise between energy consumption and the quality of the obtained image. Their analysis is drawn from the results of measurements of the current consumption for each state: standby, sensing, processing, connection, and communication. Model-based design exploration of wireless sensor node lifetimes by D. Jung, T. Teixeira, A. Barton-Sweeney, and A. Savvides, analyze how different operation modes [13], such as duty-cycle mode and event-driven mode, affect the lifetime of a camera node.

In the above literature survey the different authors have explained about the different issues in visual sensor network. None of the work mentioned above consider energy consumed with and without processing. In this paper, we deal with the energy consumed during subtraction, transmission and also during reconstruction and also calculated the image quality when the image is reconstructed. 


\section{Data Processing}

In this paper the background subtraction technique is used to reduce the energy consumption and reconstructed the original image at the server side. The analysis is carried out w.r.t energy consumption and processing time at client and server.

\subsection{Functional Block Diagram}

Figure.1 shows the functional block diagram for implementing the visual sensor network in surveillance system. We assumed that there is a visual camera which continuously monitors the area. When any object comes in the area of interest then it collects the visual data and forwards it to the server. The person sitting at the server will be in a position to monitor the remote place.

Consider a simple scenario where, camera $\mathrm{C} 1$ is captures images and sent to a server $\mathrm{S}$ for analysis. The paths are represented with an arrow in Figure 1. The motion is detected using background subtraction with some threshold value. If the subtraction is greater than threshold then motion is detected. The frames are subtracted and then sent to the server. The energy required during subtraction and transmission is calculated. The images are transmitted using TCP/IP protocol. The images are reconstructed at the server side and the energy consumed during reconstruction is also calculated.

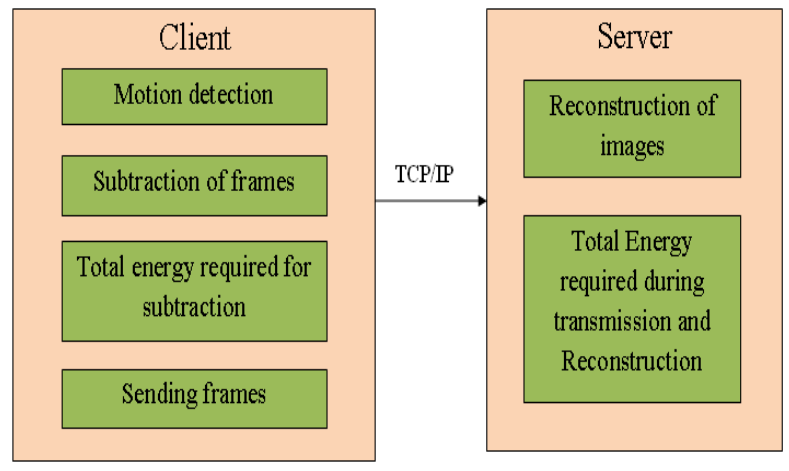

Figure 1: Functional Block Diagram for single client and single server

\subsection{Motion Detection and Overlapping of Frames}

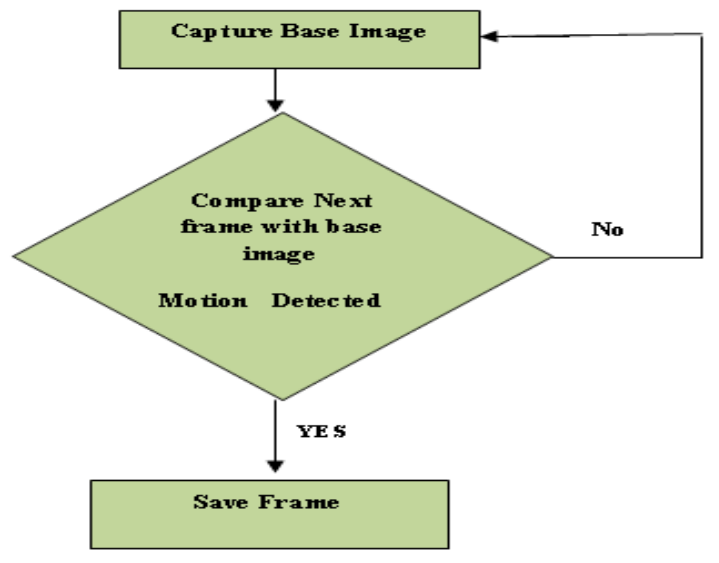


Figure 2: Overview of motion detection

The method of moving object detection is based on background subtraction for real time moving objects. The moving regions are detected by subtracting the current image pixel by pixel from a reference background image. If the pixel difference is above the threshold then it is classified as foreground otherwise it is background. Suppose if there is a change in the image then the image is sent to the server. The most commonly used approach for foreground detection is to check whether the input pixel is significantly different from the corresponding [4] background estimation $\left|\mathrm{I}_{\mathrm{t}}(\mathrm{x}, \mathrm{y})-\mathrm{B}_{\mathrm{t}}(\mathrm{x}, \mathrm{y})\right|>\mathrm{T}_{\text {threshold }}$. The absolute differences between the foreground and background image are considered.

To find the overlapping of image, we consider the difference between the foreground as well as the background image. There are two methods to find the overlapping of image - static and dynamic. In static, we compare the background with all the other frames. In dynamic we compare the consecutive frames. To calculate the overlapping of image, initially we have to consider that both images are of the same size. i.e. $320 * 240$, i.e. rows $=320$, columns $=240$. Then subtract the two images using absolute difference. If the difference is less then threshold then increment the sum. After calculating the sum the overlapped image is calculated using the formula: Overlapped image $=\operatorname{sum} /(320 * 240) * 100$.

\subsection{Reconstruction of Image}

Optimal extraction of the underlying quantities from measured data requires the removal of measurement defects such as noise and limited instrumental resolution. When the underlying quantity is an image, this process is known as image reconstruction (sometimes called image restoration, if the data are also in the form of an image).

Initially base image is sent to the server and later all the other frames are sent to the server by subtracting the base image with the other frames. This reduces the image size which in turn reduces the energy required to transmit the image. Here it shows that energy required to transmit without processing is more than with processing the image. At the server side the addition of background image and the subtracted image are processed so as to get the original image. During reconstruction of original image some noise can be seen. To reduce the noise the median filter is applied. This improves the quality of image. In the implementation part we can see the comparison between the original image and the reconstructed image.

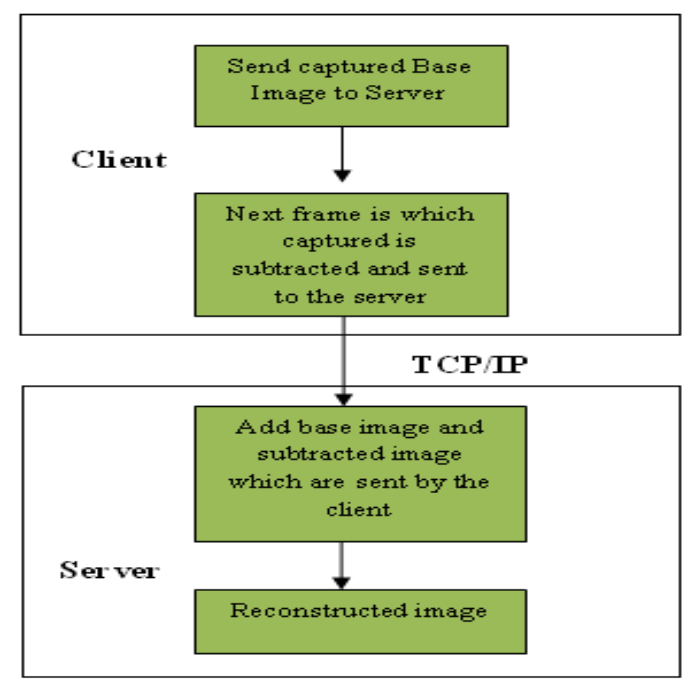

Figure 3: communication between client and server 
Algorithm for Subtraction

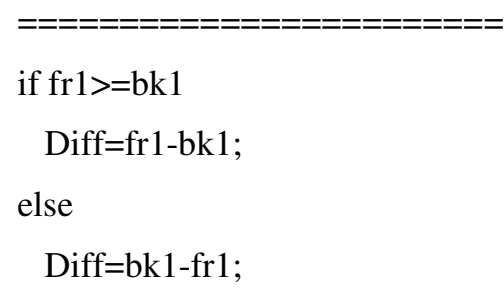

Algorithm for Reconstruction

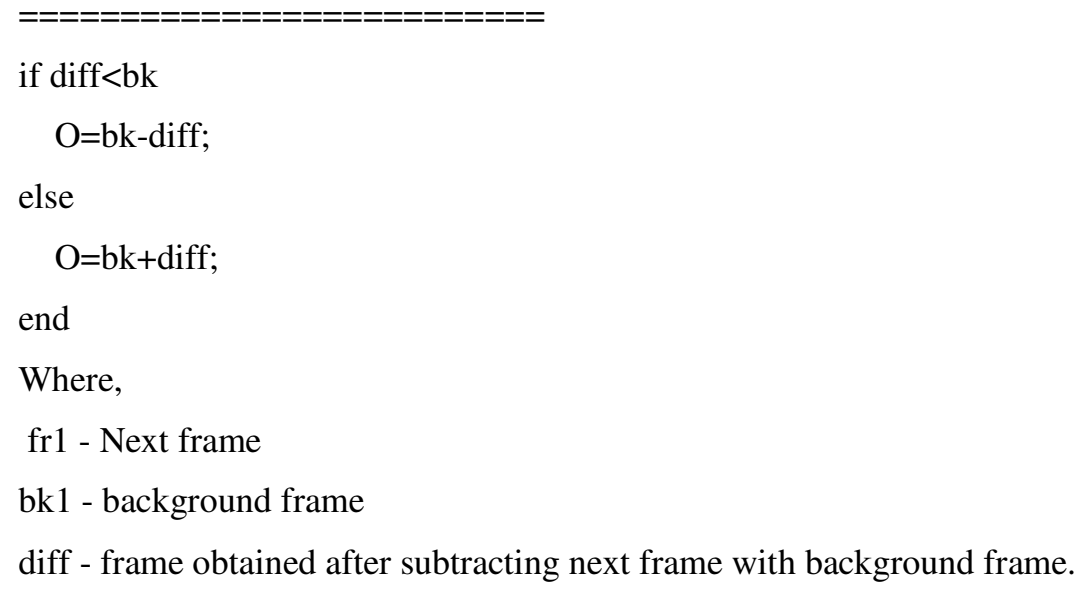

\section{IMPLEMENTATION}

Once the frames are captured at the client side they should be sent over the network [6] to the server computer. While transmitting to the server, the energy utilization must be reduced which is done using the background subtraction technique.

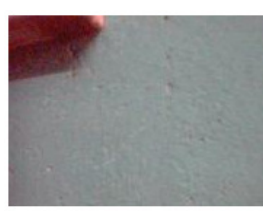

Backgraund Frame

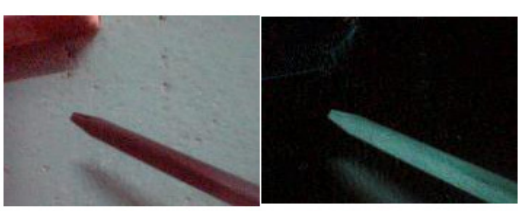

Subtracted Frame

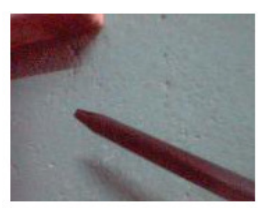

Recanstructed Frame

Figure 4: Show Reconstructed Image at the server

The quality of original and the reconstructed image using some of the parameter such as Mean Square Error, Peak Signal to Noise Ratio, MNormalized Cross-Correlation, Average Difference, Structural Content, Maximum Difference, Normalized Absolute Error, and Laplace Mean square error are used. 


\begin{tabular}{|l|c|}
\hline Mean Square Error & $M S E=\frac{1}{M N} \sum_{j=1}^{M} \sum_{k=1}^{N}\left(x_{j, k}-x_{j, k}^{\prime}\right)^{2}$ \\
\hline Peak Signal to Noise Ratio & $P S N R=10 \log \frac{\left(2^{*}-1\right)^{2}}{M S E}=10 \log \frac{255^{2}}{M S E}$ \\
\hline Normalized Cross-Correlation & $N K=\sum_{j=1}^{M} \sum_{k=1}^{N} x_{j, k} \cdot x_{j, k}^{\prime} / \sum_{j=1}^{M} \sum_{k=1}^{N} x_{j, k}^{2}$ \\
\hline Average Difference & $A D=\sum_{j=1}^{M} \sum_{k=1}^{N}\left(x_{j, k}-x_{j, k}^{\prime}\right) / M N$ \\
\hline Structural Content & $S C=\sum_{j=1}^{M} \sum_{k=1}^{N} x_{j, k}{ }^{2} / \sum_{j=1}^{M} \sum_{k=1}^{N} x_{j, k}^{\prime}{ }^{2}$ \\
\hline Maximum Difference & $M D=M a x\left(\left|x_{j, k}-x_{j, k}^{\prime}\right|\right)$ \\
\hline Laplacian Mean & $L M S E=\sum_{j=1}^{M} \sum_{k=1}^{N}\left[O\left(x_{j, k}\right)-O\left(x_{j, k}^{\prime}\right)\right] / \sum_{j=1}^{M} \sum_{k=1}^{N}\left[O\left(x_{j, k}\right)\right]^{2}$ \\
\hline Normare Error & $O\left(x_{j, k}\right)=x_{j+1, k}+x_{j-1, k}+x_{j, k+1}+x_{j, k-1}-4 x_{j, k}$ \\
\hline
\end{tabular}

Table 1: Table shows the formula to calculate the quality of image

\begin{tabular}{|l|c|l|c|c|c|c|c|c|}
\hline Images & MSE & PSNR & MNCC & AD & SC & MD & NAE & LMSE \\
\hline $\begin{array}{l}\text { Original } \\
\text { frame }\end{array}$ & 0 & 99 & 1 & 0 & 1 & 0 & 0 & 0 \\
\hline $\begin{array}{l}\text { Reconstructed } \\
\text { frame }\end{array}$ & 12.9717 & 37.0008 & 0.9913 & 1.1095 & 1.0169 & 76 & 0.0134 & 0.0387 \\
\hline
\end{tabular}

Table.2: Table shows the values of original frame and the reconstructed frame

\begin{tabular}{|c|c|c|c|c|c|c|}
\hline \multicolumn{3}{|c|}{ Subtraction } & \multicolumn{2}{c|}{ Transmission } & \multicolumn{2}{c|}{ Reconstruction } \\
\hline Overlapping & Time in ms & $\begin{array}{c}\text { Energy in } \\
\mathrm{mJ}\end{array}$ & Time in ms & Energy in mJ & $\begin{array}{c}\text { Time in } \\
\mathrm{ms}\end{array}$ & $\begin{array}{c}\text { Energy in } \\
\mathrm{mJ}\end{array}$ \\
\hline $0 \%$ & 0.046 & 0.1432 & 8.39 & 15.11 & 0.9023 & 1.6253 \\
\hline $50 \%$ & 0.0625 & 0.3133 & 7.7343 & 13.932 & 0.0813 & 0.1464 \\
\hline $100 \%$ & 0.4687 & 11.7838 & 7.625 & 13.735 & 0.0592 & 0.1066 \\
\hline
\end{tabular}

Table 3: Shows the time and energy required for different percentage of overlapping Frame

\section{Result AND Discussion}

The experiments were performed for different resolution in Windows Application Lab with camera deployed and connected to computer. The camera specification is 3.5 mega pixel and focal length $=3.85 \mathrm{~mm}$. Figure 5 shows that the motion detection depends on the resolution of the camera. As the resolution is more the sensitivity to detect the motion is more and the angle or the field of view is 30 degree. 
International journal of computer science \& information Technology (IJCSIT) Vol.2, No.5, October 2010

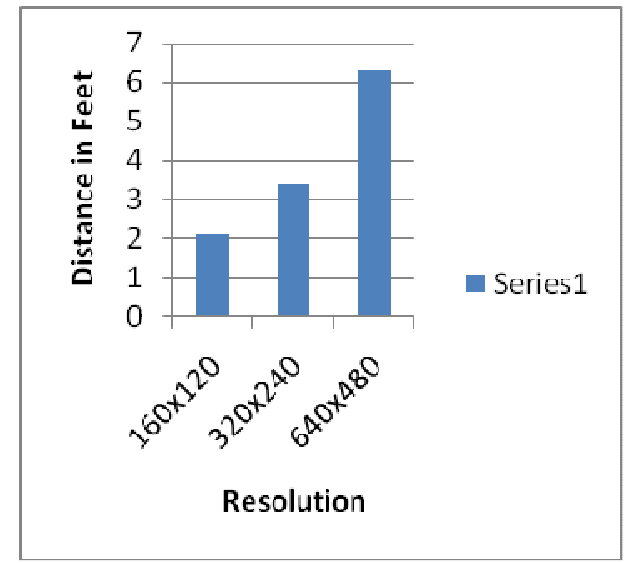

Figure 5: Resolution versus Distance

Figure 6 also indicates the energy used while transmitting the Frame with and without processing.

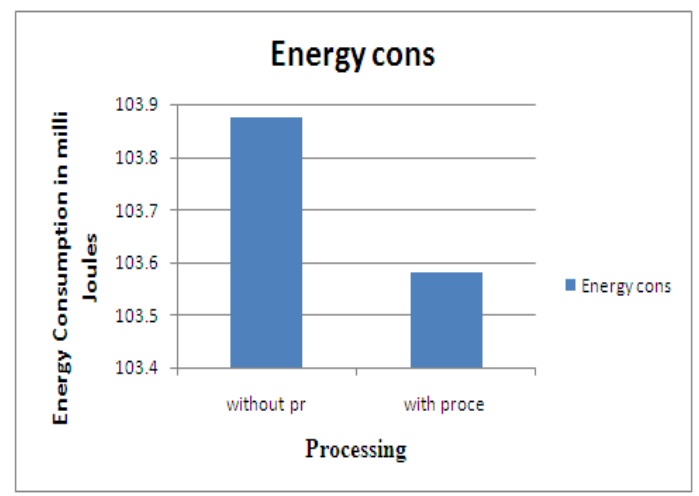

Figure 6: Frame versus Energy consumption

Figure 7 shows the bandwidth utilized during transmission of the Frame with and without processing.

\section{Bandwidth $($ Mbits $)=$ FrameSize $(\mathrm{Kb}) *$ FPS $*$ Cameras $*$ 8 / 1024}

The above formula is used to calculate the bandwidth utilized and the resulting graph is plotted as in Figure 7.

Frame per second according to camera specification $=15 \mathrm{fps}$

Cameras used $=1$

Frame Size in $\mathrm{Kb}$ 


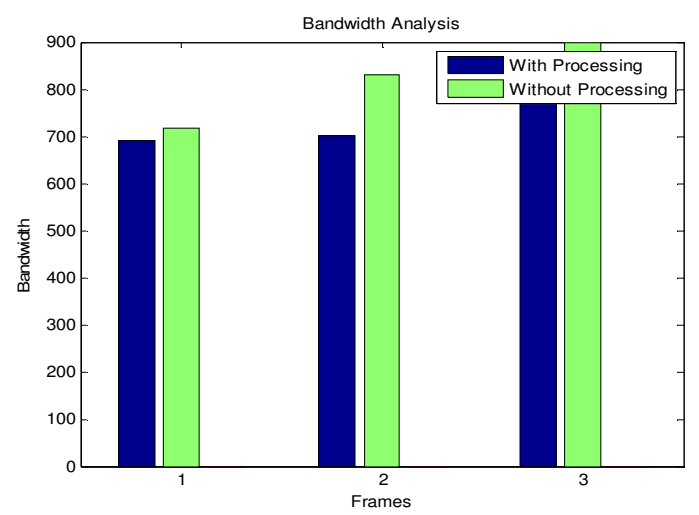

Figure 7: Frame versus Bandwidth utilized

\section{CONClusion}

This paper presents and discusses the analysis with respect to resolution of camera and sensitivity to detect motion. We have seen that as the resolution is more the sensitivity to detect the motion is more. It is observed that the performance of processing with image in terms of energy consumption is efficient as compared to without processing the image, which in turn reduces the bandwidth utilization.

\section{REFERENCES}

[1] D. Estrin, D. Culler, K.Pister, and G.Sukatme, "Connecting the Physical World with pervasive Networks,” IEEE Pervasive Computing, pp. 59-69, 2002.

[2] Kit- Yee chow, King-Shan Lui and Edmund y. Lam "Balancing Image Quality and Energy Consumption in Visual Sensor Networks "IEEE 2006.

[3] S. Nath, P. B. Gibbons, S. Seshan, and Z. R. Anderson, "Synopsis Diffusion for Robust Aggregation in Sensor Networks," ACM Sensys, 2004.

[4] Sen-Ching, S. Cheung and Chandrika Kamath "Robust Techniques for Background Subtraction in Urban Traffic Vedio" center for applied scientific computing lawrence livermore national laboratory 7000 East Avenue, Livermore, ca 94550.

[5] Ypussef Charfi, Bell Canada Naoki Wakamiya and Masayuki Murata, "Challenging Issues In Visual Sensor Networks", Osaka University, 2009.

[6] G. J. Pottie and W. J. Kaiser, "Wireless Integrated Network Sensors," Communications of the ACM, vol. 43, no. 5, pp. 51- 58, 2000.

[7] B. Krishnamachari, D. Estrin, and S .Wicker, "The Impact of Data Aggregation in Wireless Sensor Networks," International Workshop of Distributed Event Based Systems, pp. 575-578, 2002.

[8] H.Wu and A.A. Abouzeid, "Power Aware Image Transmission in Energy Constrained Wireless Networks," IEEE symposium on computers and communications (iscc), pp. 202- 207, 2004.

[9] H.Wu and A.A. Abouzeid, "Energy Efficient Distributed JPEG2000 Image Compression in Multihop Wireless Networks," workshop on applications and services in wireless networks (aswn), 2004.

[10] M. Wu and C. W. Chen, "Multiple Bitstream Image Transmission over Wireless Sensor Networks," in proceedings of 2D IEEE International Conference on Sensors, vol. 2, pp. 727-731, Toronto, Canada, October 2003. 
International journal of computer science \& information Technology (IJCSIT) Vol.2, No.5, October 2010

[11] King-Shan Lui and Edmund Y. Lam, "Image Transmission in Sensor Networks," workshop on Signal Processing Systems (sips), 2005.

[12] Deokwoo Jung “A MATLAB " Wireless Sensor Node Platform Lifetime Prediction \& Simulation Package Version 2.0.0 “ User's manual November 15, 2007.

[13] D. Jung, T. Teixeira, A. Barton-Sweeney, and A. Savvides, "Model-based design exploration of wireless sensor node lifetimes," in Proceedings of the 4th European Conference on Wireless Sensor Networks, pp. 277-292, 2007.

[14] J. C. Dagher, M. W. Marcellin, and M. A. Neifeld, "A method for coordinating the distributed transmission of imagery," IEEE Transactions on Image Processing, vol. 15, no. 7, pp. 1705- 1717, 2006.

[15] S. Hengstler, D. Prashanth, S. Fong, and H. Aghajan, "Mesh- Eye: a hybrid-resolution smart camera mote for applications in distributed intelligent surveillance," in Proceedings of the $6^{\text {th }}$ International Symposium on Information Processing in Sensor Networks (IPSN ’07), pp. 360-369, 2007.

[16] D. Devarajan, R. J. Radke, and H. Chung, "Distributed metric calibration of ad hoc camera networks," ACM Transactions on Sensor Networks, vol. 2, no. 3, pp. 380-403, 2006.

\section{Authors}

Asst. Prof. Harish H. Kenchannavar received his Masters degree (M.E) from Shivaji University, Walchand College of Engineering \& Tech, Sangli, Maharashtra. He has registered for Phd degree under Visvesvaraya Technological University, Belgaum. Research Center:- SDMCET, Dharwad, Karnataka under the guidance of Prof. Umakant P. Kulkarni. He is currently working as Assistant Professor in Computer Science Department, Gogte Institute of Technology, Belgaum, Karnataka. He has presented and published a papers at national and IEEE International conferences. His research interests include computer networking, simulation and modeling, visual sensor networks and queuing algorithms.

Miss Sushma S. Kudtarkar doing her Masters degree (M.Tech) from Gogte Institute of Technology, Belgaum, Karnataka. She is currently working as a Lecturer in Electronics and Communication Department, Shaikh College of Engineering and Technology, Belgaum, Karnataka. She has presented and published a paper at IEEE International conferences. 
International journal of computer science \& information Technology (IJCSIT) Vol.2, No.5, October 2010

Prof. Umakant P. Kulkarni received his $\mathrm{PhD}$ degree from Shivaji University, Kolhapur. Research Center:- Walchand College of Engineering \& Tech, Sangli, Maharashtra in Nov 2007 . He has completed his Masters degree (M.E) from PSG, Coimbatore. He is currently working as Professor in Computer Science Department, SDMCET, Dharwad, Karnataka. He has published and presented a number of papers in many reputed journals and at International IEEE conferences as well. This apart he also has presented technical talks and tutorials on various aspects of Mobile Agents. His research interests include mobile agents, computer networking, and distributed networking. 